\title{
To Be or Not to Be - A Research Subject
}

\author{
- Eric M. Meslin and Peter H. Schwartz
}

For most people, the terms clinical trial, institutional review board, riskbenefit assessment, and informed consent make as much sense as the instruction manual for a complicated piece of technology. Indeed, most people probably do not know that when medical research is conducted to test a new medicine, there are rules and procedures the scientists must follow to ensure that the research is both scientifically sound and ethically safe. Most people do not know there are different kinds of medical studies; some are conducted on people who already have a disease or medical condition, and others are performed on healthy volunteers who want to help science find answers. Yet both types of potential research subjects have many of the same questions, the answers to which can help them decide whether to participate in the study, what their rights are, and what decisions they may have to make. This chapter reviews the basics of being a research subject - including the potential benefits and risks of making this important decision.

\section{The Basics}

Once you become a patient, it won't be long before somebody asks you to be a research subject. For example, your doctor might want 
to enroll you in a clinical trial of a new medication, possibly for a condition for which other drugs are available - such as high blood pressure - or for one for which there are no approved treatments yet. Other research studies do not involve a medication or treatment at all; many just ask you to fill out a questionnaire or give a blood sample.

Of course, patients aren't the only people approached to participate in medical research. Many projects involve healthy people. In fact, the earliest stages of research on any new medication almost always involve testing in healthy people to determine side effects and correct dosages. Medical research is always going on all over the world, and there are so many different types of research, it may be hard to make any reliable generalizations. Even if you have never been a research subject, the effects of research certainly have an impact on your everyday life.

No matter what sort of research you are invited to participate in, or whether you are a patient when you are asked, it's entirely up to you whether or not to do it. This decision is important and may have many implications for your health and well-being, as well as those of other patients now and in the future. Making a good decision - the right one for you - requires you to become educated about topics you may not have thought about before, some of which may be quite complicated. This chapter explains the key issues to help you make a good decision.

\section{What Is Research?}

The first step in deciding whether to participate in a research study is to understand what research is and how it differs from medical treatment. According to some of the most important regulations, research is any "systematic investigation ... designed to develop or contribute to generalizable knowledge" (common rule). For a project to count as medical research, the knowledge involved must be related to the effort to improve health and medical care. Of course, this endeavor encompasses a great variety of activities. Some discussions of research emphasize that it extends from the bench to the bedside, that is, from basic studies on molecules and animals in a laboratory all the way to clinical research conducted on humans to determine the effect of new medications and procedures. The sort of research you will be invited to participate in is called human subjects research because it involves human beings (you and others). Human subjects research is governed by special regulations and ethical principles and guidelines, which are discussed in this chapter. 


\section{Should I Be Concerned about Unethical Research?}

Research involving human beings has a long and complex history. Although physicians and scientists have been studying the body for millennia, only in the past fifty years has research involving human beings been a major activity of universities, industry, and even nations. At the same time, an entire system for protecting human subjects from harm was developed in the United States and around the world, partly as a result of many cases of unethical research that came to light. These cases include the horrific concentration camp experiments conducted by the Nazis, some of which involved the torture and murder of many people. However, although those experiments were at the unethical extreme, many other cases of ethically problematic research occurred after World War II in prominent U.S. universities and were sponsored by the federal government. For example, the Tuskegee study that ran from 1932 until it was finally stopped in 1972 observed the progression of syphilis in poor black men in rural Alabama. In this study, researchers kept the men unaware of their infection and even took steps to make sure they did not receive medical treatment. Other studies, such as the Willowbrook State School experiments, in which children were intentionally exposed to hepatitis, and the Jewish Chronic Disease Hospital study, in which elderly patients were injected with cancer cells, resulted in public outrage and concerted action by the U.S. Congress to develop a comprehensive system of rules, regulations, and procedures to make sure all medical research is conducted ethically. Although the rules are quite complex, they involve three basic assumptions any person thinking about participating in a research study should know about.

1. All subjects must give "informed consent" before participating in a research study.

2. The risks to any humans participating will be minimized as much as reasonably possible, and a study should be performed only if the possible benefits outweigh the risks.

3. All studies conducted on human beings must have gone through an independent committee for review and ethical approval.

Research is a serious endeavor and is not an activity scientists undertake lightly. In many cases, researchers are funded to carry out studies with grants from federal research organizations such as the National Institutes of Health and the National Science Foundation - organizations supported by taxpayers. In fact, to receive these grants, researchers are 
legally bound to follow ethics regulations, including the requirement to obtain informed consent.

\section{What Is Informed Consent?}

Before you enroll in a research project as a research subject - before you undergo any tests or interventions - you must agree to it. Your agreement is called "informed consent" because you must be informed about the study so you can decide whether you want to join and you must give consent (approval) before anybody does anything to you. Some of the issues you must be informed about include the following three questions, which you should keep in mind:

1. Why is this study being done in the first place? Most researchers are committed to finding answers to health problems. So, it matters why a study is being done - is it just a frivolous wild goose chase or is it a serious search for the answer to a problem that might one day lead to a treatment or cure for a disease?

2. What exactly will happen to me? This is an obvious question, but the details are important. You should know whether your involvement consists of one visit or many visits, whether it involves an invasive procedure like a blood draw or something even more invasive, whether you will be answering surveys, and whether people will be contacting you for more information. The more you know about the actual study and what you will experience, the more comfortable you will be with your decision.

3. What are the risks and potential benefits to me? Knowing what will happen to you is not the same as knowing what the risks and potential benefits are to you from participating. Consider the idea of "benefits" first. It is common for patients to believe that if a doctor asks them to participate in research, they will receive medical care that will benefit them. This is not a good assumption for a couple of reasons. First, it makes sense to study a test or treatment for some condition only if there is uncertainty over whether there are real benefits from it. Second, although it is possible for a patient involved in a research study to get better treatment, research studies are not designed with this intention at all.

The goal of medical research is to obtain information that can improve health and medical care in the future, the sort of "generalizable knowledge" mentioned in the definition given earlier. For instance, many studies attempting to determine the effect of a medication include one group of subjects randomly assigned to receive no treatment at all (a placebo). (We'll say more about this later.) In considering whether to participate in a study, it is important to get a 
clear picture of what benefits you may receive, if any, as well as the benefits that may accrue to others.

Of course, the risks you will face are just as important as the benefits and depend on the type of research project in which you are asked to participate. If you are going to be given an experimental medication or undergo any procedures, the researchers must provide you with information about any possible side effects or complications. Even if the research project does not involve any medications or procedures - for instance, if you are just answering a questionnaire the researchers must describe any negative effects there could be for you (e.g., a problem with confidentiality, i.e., someone getting information about you). The information the researcher gives you about risks may be very complex, and we'll also say more about how to handle that information later in the chapter.

Overall, there are many other things you might be told - and should be told - however, at the very least, the three key questions described earlier should be answered to your satisfaction. Moreover, these and other issues usually are detailed in a form (the informed consent document) that you will receive, read, and need to sign before proceeding. Remember, if you have learned about the study and you decide not to participate, that is your right too. No one can (or should try to) force you to participate in research. It's your choice.

\section{Research on Those Who Can't Consent for Themselves}

There are two situations in which you may be asked to give consent for research conducted on someone else. One situation involves children. Because children cannot give legal consent to participate in research, their parents or guardians must give consent before they can be enrolled. A child may be given some information, based on his or her ability to understand, and asked whether he or she would like to participate (the child's agreement is called assent); however, his or her parent or guardian must provide the informed consent. The other situation involves people who cannot consent for themselves because they have a mental illness, such as dementia. In this case, as with research involving children, the person's legal guardian must give consent before he or she can be enrolled in the study. The regulations concerning this type of research are particularly stringent and attempt to ensure that risks are minimized and the study truly is worth doing. If you are making a decision about research participation in one of these situations - for a child or another adult - you are expected to 
represent the best interests of that person, deciding for them as if you were deciding for yourself.

\section{How Is Research Regulated and Reviewed?}

Even before researchers start a project, and before inviting anybody to participate as a research subject, they must obtain the approval of a committee of experts who have reviewed the study in detail, including the informed consent document. In the United States, this committee is called an institutional review board (IRB). There are more than 3,500 IRBs in the United States located at medical centers, universities, and research institutions. In fact, the U.S. government requires any human subjects research study performed at an institution that receives federal grants or contracts to be reviewed and approved by an IRB. IRBs are made up of doctors, nurses, other researchers, community representatives, and other members who examine a range of documents the researchers must submit before they can go forward with their study. Again, the regulations the IRB applies are quite detailed, but they distill to the three general principles listed earlier: 1) informed consent, 2) minimizing risk to subjects, and 3) ensuring the benefits outweigh the risks. IRBs also review ongoing studies to make sure they are complying with regulations and with their previously stated plans.

Most IRBs are based at the institution, such as a university, where the research is being conducted; however, there are now some cases in which the IRB for a study is in a location other than the one where the study is being done. For instance, one IRB may oversee research being conducted at several universities or clinics. When you are approached to participate in a research project, it is perfectly reasonable to ask which IRB approved the study and how you can contact the board members if you have any questions. In rare cases, a research project may go forward without IRB review - for example, if it is being run by an organization that receives no federal grants or contracts. Therefore, you may want to make sure there was an IRB or other similar review of the study you are being invited to join. We think you should be somewhat skeptical of any study that was not approved by an IRB, especially if there is any risk to you from participating.

Besides IRBs, there are other bodies that review certain types of medical research. For example, if the project involves a new medication, the researchers must get permission from the Food and Drug 
Administration (FDA) to perform the study. To get FDA approval, the researchers must show evidence that the drug to be studied has acceptable risks and real promise, based on data from laboratory and/or animal studies. Many universities have special bodies that review certain kinds of research - such as cancer or gene therapy studies - again, it is perfectly reasonable to ask which groups reviewed a project before you agree to participate.

\section{What Type of Research Project Is This?}

Although the issues we discuss in this chapter apply to all sorts of medical research, important differences exist among the various types of studies you might be invited to join. Therefore, you should know a little about each kind of research project so you understand the type of research involved, or at least the terminology being tossed around, in any study in which you might participate.

\section{Clinical Drug Trials}

In some ways, clinical drug trials are the prototypical research studies. Clinical drug trials are studies of new medications, or new uses for existing ones, to determine whether they can be used to treat specific diseases or other medical problems. If you are being invited to participate in a clinical drug trial, you should be aware of some specific issues and questions.

In what stage of testing is the drug? All drugs go through (at least) three stages of testing: phase I, phase II, and phase III. Phase I trials occur first and focus almost entirely on identifying the proper dosage for the medication as well as any side effects. These studies often are designed to begin with a very low dosage of the drug and then to increase it slowly until serious side effects are seen. Phase II trials focus on determining whether the treatment actually will help patients. These studies usually involve a smaller number of patients who receive the medication and have their responses to it measured carefully. Phase III trials are the large randomized, placebocontrolled studies that help clarify exactly how much benefit the drug has in treating the disease in question, whether it truly is safe, and whether it is an improvement over other medications used to treat that illness.

The take-home message is that if the study is phase I or phase II, there is relatively little evidence the treatment actually is beneficial 
and/or any better than other available treatments. In fact, the reason the researchers are doing the study is to look for that evidence. Therefore, you should be somewhat skeptical of any implication that a medication probably will help you; if the researcher knew that, they would not be doing the study. In fact, the IRB should refuse to let the study go on! The findings of a phase I study may be promising or give the study investigators hope, but, again, no one knows what future research will discover. By phase III, more evidence will evolve that the study investigators can use to explain the reasons for their hope, as well as any risks.

Will everybody get the treatment, or is there a control group? In many clinical trials (especially phase III), not all subjects receive the study drug; some get no treatment (a placebo) or the currently accepted treatment. Therefore, if you are participating in a study to get a new treatment that has some promise, you should know whether there is a chance you won't get the study treatment. In many cases, neither the subjects nor the researchers know who is getting the experimental treatment and who is getting the placebo or standard treatment. This technique ensures that the subjects in the two study groups are not treated differently, which could bias the results.

\section{Other Clinical Trials}

Some clinical trials are designed to study a medical procedure or device, rather than a drug. For example, one study may compare two different ways of implanting a cardiac stent; another may compare two methods of repairing torn knee cartilage. As with clinical drug trials, clinical trials of procedures or devices raise several ethical issues, require a high level of regulatory scrutiny, must be reviewed and approved by IRBs, and cannot take place in humans without their informed consent. With regard to participation in this type of trial versus a clinical drug trial, many of the same questions arise: How much testing has been done so far on the benefits and risks of the test or procedure compared with the standard one? What benefits or risks have been seen so far? Is there a control group, or do all study participants get the experimental intervention?

\section{Observational Research}

Some studies are performed not to test a treatment or procedure, but to monitor people receiving a certain treatment. Investigators in these 
studies record the participants' treatment and their response to it, talk to and/or examine the subjects, and may look at their medical records during and after treatment. If you participate in this sort of trial, called an observational study, the main discomfort or risk to you might be limited to blood tests or x-rays. Such studies also are important for improving medical care - by allowing researchers to figure out what is or is not working - and they involve much less risk than many clinical studies.

However, as with clinical trials - and all research studies, for that matter - you should think about the questions listed earlier regarding what exactly will happen to you and what the benefits and risks are to you. Don't accept vague answers: everything should be spelled out to your satisfaction. If someone refers to a particular medical test or x-ray you are unsure about, you have every right to ask for more information, including exactly how long the procedure takes and how uncomfortable it is.

Another risk of observational studies - and any study in which the researchers need to keep track of information about the participants is the possibility someone will learn private information about you and inadvertently disclose it to someone else who is not authorized to have it. Privacy and confidentiality are important values in our society, so it is especially important for you to be aware of the plans in place to protect your privacy and confidentiality. The sort of information researchers collect is personal and may be related to your medical problems, your treatment, or any answers you provide on a survey. The researchers are obligated to keep this information private and to prevent anybody else from accessing it. Like banks, study investigators use various techniques - locked computers, passwords, and encrypted databases - to keep your information as safe as possible; however, sometimes there is a breach. The informed consent document must list this risk and describe how your information will be kept safe.

Of course some studies can be conducted without requiring researchers to collect personal information about you. Some research, such as public health surveillance studies or studies involving blood samples or other biological material, can be carried out without linking the research findings to a particular person. Studies involving anonymized data of this kind pose no specific risk to individuals.

\section{Survey Research}

In some studies, the researchers want nothing more than to speak to the participants and/or have them fill out questionnaires. If you 
participate in one of these surveys, basically your only important risk is any loss of confidentiality. In fact, this type of study carries so little risk, the researcher may approach you without asking you to read and sign an informed consent document and will ask for your verbal consent only when he or she is talking to you. Generally, an IRB still has to approve this type of study, but based on the fact that the risks are so low, there is no need to write everything down. However, you still are entitled to ask for as much information as you want. Again, it is up to you whether you want to take the time and effort to help, and there is no reason you should feel you have to do so. As always, participation in research is basically an altruistic action for the good of people other than yourself - and you should feel good about volunteering if you decide to participate. There may or may not be some small token of gratitude (e.g., a gift card in a small denomination) to thank you for your time, and the researchers should act graciously toward you for helping them with their project and (hopefully) scientific advancement.

\section{Biobanks}

Finally, a special kind of observational research becoming more and more common is called biobank research. In biobank studies, researchers collect medical information from subjects and match it with biological data, such as blood or tissue samples or information from specific examinations (e.g., a physical exam or an x-ray result). Sometimes the tissue is left over from a medical procedure, such as a breast biopsy, and the researchers want the subject's permission to keep that extra tissue instead of throwing it out. Sometimes, the researchers want permission to call participants regularly to get updates on their medical history and even to look at their medical records at regular intervals. Such biobanks aim to collect and store information from hundreds to millions of people to find out more about how our genes and other facts about us affect our health and life.

As with all other research, if you are invited to join a biobank study, you should feel good about asking as many questions as you want to find out exactly what will be done and what the benefits and risks are to you. As in observational studies, the main risks to you are those associated with any procedure the researchers propose to do to collect the sample or conduct the exam, including a blood draw or other testing. In addition, because the biobank will be in place possibly for many years, the researchers must address what they will do to protect your information from unintended disclosure (i.e., to avoid loss of confidentiality). They also should explain a bit about who will use the 
biobank, how your information might be used in future studies, and how projects will be chosen in the future.

\section{More Specifics and Complexities}

The previous discussion is just an overview of research, the regulation of research, and the choices you will face if you are invited to participate in a research study. Hopefully, it will get you thinking about what to ask and what to consider if and when you are approached. However, for each of the topics described earlier, the issues get somewhat more complex when you look at them more closely. It is important for you to be aware of these complexities as you decide whether to participate in research.

\section{When Medical Research Can Be Confused with Treatment}

Sometimes participating in a research study may seem like receiving medical treatment, but, as we emphasized earlier, they are very different. They may seem similar because the procedures used in research (e.g., surveys, blood draws, administration of medicine) are typical health care procedures. Moreover, the interactions may feel the same with a doctor and nurse talking to you about your medical history, symptoms, medications, and so forth - and may even occur in a doctor's office or hospital.

However, the two activities - medical treatment and medical research - are not the same. When you see your doctor, he or she has the primary goal and responsibility of protecting and/or restoring your health by treating any illness. In contrast, the primary goal and obligation of the researchers in a study (and any doctors and nurses who are involved) is to the research project. However, they also must watch out for your health and well-being, and there are many protections in place to watch over you while you are participating in the project. For example, in some clinical drug trials, there is a separate committee called a data safety and monitoring board that reviews the study on an ongoing basis. This committee has the authority to stop the study if it believes that patients might be harmed. However, the researchers' goals in doing the study, and their interactions with you as a research subject, are designed to increase scientific and medical knowledge and further (soon or someday) the ability to prevent or treat disease. The reason for doing the research is not to restore or improve your health. 
So, let's go back to considering the benefits you might receive from participating in research. In some cases, the possible benefits to you might be very specific. For example, a drug not yet approved for use in medical treatment is being studied for a condition you have, and it already has shown some promising results. Your doctor may feel this treatment is the best one for you, even though it is unproven; thus, he or she may recommend that you enroll in a study of that medication. This sort of situation comes up often for patients with advanced cancer for whom all approved treatments were tried and failed to cure or control the cancer. At this point, with no other approved treatment available, doctors may recommend, or even encourage, these patients to enroll in a trial of some new medication.

In short, these patients are gambling that the new medication will be successful, whereas the drugs already studied and approved have failed; as such, their decision to enroll in the study seems more like a search for a new treatment than participation in research. However, it is important to understand that because the medication is being provided as part of research, the criteria for who gets the medication, the way it is given, and the sort of decisions the doctor or care team can make are driven by the study design, not by an effort to maximize patient care.

Many people looking at the ethics of research and informed consent have noticed that when patients in this type of situation are offered a research medication as part of a study, they may overestimate the chance that the medication will actually help. It may be that the studies of the medication are in very early stages, and any possible benefit is completely unclear. In phase I studies, in which the goal is to determine the proper dose to use for future testing, there is little likelihood of benefit to any particular person. In other studies, such as phase III clinical trials, there is a group of subjects who get no treatment at all (the placebo group) so that the researchers can better determine the effects of the medication on people; in many cases, the research subjects, and even the researchers, do not know (until the end of the study) who got the research medication and who got the placebo.

In short, if you are thinking about participating in a research study because you think you will get a specific benefit - such as a medication you cannot get any other way - you should determine what the chances are that you will actually get the medication and that it will actually help you. In the end, whether to enter a study is a decision you should make mostly based on a desire to help advance science, as we discuss next. 


\section{Should I Participate in Research to Feel Good or Make Money (or Both)?}

The differences in the nature of medical research versus that of medical treatment mean there are important differences in the motivation for participating in one or the other. When you agree to participate in a research project, you are doing it primarily for the benefit of others. The people who benefit from the knowledge gained from the study may have the same disease or condition you have or may belong to future generations. Or, your participation may be a contribution to increasing doctors' abilities to prevent or treat disease. These are important reasons for participating, but they are also very different from your reasons for getting medical treatment, in which the main goal should be to protect or recover your own health. Simply put, the difference may be one of altruism versus personal benefit.

Of course, not everyone has altruistic reasons for participating in research. Some people find it interesting to join a study; others may participate because there is a modest stipend. If you receive payment for participation, however, you should ask yourself whether the money is what convinced you to participate. No one is supposed to participate in research just for the money; the money is meant to defray participants' expenses or reimburse them for their time and is not meant to be payment like a paycheck. Although there are no set rates for remuneration, the general principle is that as the study risk or the subjects' involvement increases, so too will the amount of money provided. For example, a one-time blood draw to look for a certain enzyme or protein is far less risky than a study of the safety of a new medicine that involves multiple doses and tests over many weeks, so it will provide a smaller stipend.

There is evidence that some people participate in research only for the money. In particular, some research is carried out in healthy individuals, and a thriving community has developed made up of people who travel from place to place signing up for these studies. Although the payments are relatively small, these people - who call the practice "guinea pigging" - can make a modest living by doing this.

\section{Informed Consent}

To think more deeply about the process of informed consent, consider the last of the three key questions listed earlier in this chapter: What are the risks and potential benefits for me? 
Consider a hypothetical study of some new medication for a condition you have. In this study, all the subjects will get the medication; make regular visits to the doctor's office, where blood will be drawn; and have an x-ray taken every 3 months for the next year. The main benefit to you in this case may be the chance that the medication can help: many cancer patients are in this situation when they are invited to join research projects studying new chemotherapy medications. Remember, however, that the whole idea of a research project is that doctors are still unsure whether the medication really works and what the possible side effects might be. Although it might be tempting to assume you will benefit, this is not certain.

Now consider the risks. Any trial of a new medication in human beings will occur only after there has been a significant amount of testing in animals and, in many cases, in at least some humans. Therefore, the researchers approaching you should be able to say something about the possible risks. Remember, though, that they will still be quite unsure of the side effects - which is the reason they are doing another study - and so they have to give you a list, which is usually long, of all the possible complications. Any of these complications might occur, they will tell you, but they can't tell you which ones. The previous research may or may not be able to give you a sense of the specific percentage of subjects who might get each side effect: Will it happen in two thirds of people taking the drug, or one third, or one in ten, or one in one hundred? Often researchers won't know, because determining these answers is one of the goals of research. Because current regulations require researchers to describe all the possible risks of an experimental medication or procedure, the informed consent document often looks like a laundry list of many, many bad things that could happen to you (but may or may not actually happen), and it is a good idea to ask how you are supposed to respond to such a form.

The first step in making sense of this form is to look at the words describing the possible complications and make sure you understand them. For example, the form may say that one possible side effect is cerebral edema, a term you may not understand. Even if it is described as "swelling of the brain," you likely would want to know more: Is it serious? Can it cause brain damage? Can any effects be reversed? A better description would be "swelling of the brain, which may cause confusion, or even coma and death." In the end, your understanding of the consent form depends on your asking the researcher to explain these terms, and you should never be embarrassed to insist on that. Because each person is different, there is no set of rules describing 
how much information must be given to explain a risk or its severity. For example, the term cerebral edema may be understandable for one person (he or she may be a physician or nurse), whereas others will need more information. In short, there is no shame in asking questions and expecting a conversation and answers.

Similar confusion may arise when the informed consent document tries to describe the likelihood of different complications occurring. Again, your understanding will depend on asking and getting answers to any questions you have. What about the description of edema as a possible side effect that "might" happen. What does might mean here? Is it a very remote possibility? A rare but real possibility? A 1 in 1,000 chance of it happening to you? A one in a million chance? Researchers and IRBs struggle to determine how much specificity is needed and whether that specificity will confuse people rather than educate them.

Try this experiment on yourself: Write a list of probability words, ranging from never to always; include as many words as you can think of, such as might, usually, sometimes, rarely, and so on. Then try to assign percentages to the words. For example, never should be $0 \%$ and always $100 \%$. But what about the other words? You might put this list together and share it with a researcher who gives you an informed consent form containing words such as might and possibly. At the very least, this will be an opportunity to have an informed conversation because you may want to know more about a study than what is described in the consent form. You should expect to have questions about things written on the informed consent form (or about anything else the researcher describes), and you should feel free to ask them. Perhaps most importantly, don't bow to the subtle pressure people sometimes use to stop you from asking more questions. The doctor or researcher may look like he or she is in a hurry, but he or she is obligated to explain things to you in as much detail as you want. Don't be shy about asking for more information; remember, they are asking you to do them the favor of participating in their study.

\section{What Happens If I Am Harmed or Injured In a Research Study?}

Although all research involves some type of inconvenience (your time and effort), not all research is physically risky. The overwhelming majority of studies conducted in the United States do not result in any serious harm or injury. In fact, when serious harm does occur, it is a source of great concern. Several years ago, when a patient died in 
a gene therapy study, the entire system for reviewing and approving these types of studies was reviewed and revised to ensure it would not happen again. Still, research by its nature is an exploration of something new, and there is always the chance something bad might happen. A good informed consent document outlines the possible risks (both their severity and likelihood) clearly, letting you know what you can expect. However, another purpose of the consent form is legal - by informing you of the possibility of certain risks, your agreement to participate means you are prepared to accept the possibility that one or more of the harms might happen to you. Some of these may be minor, whereas others may be more significant. That's why it is such an important decision to participate and to be fully informed.

It is equally important for the researchers (and the consent form) to tell you what will happen if you do experience one of the identified risks. Will they treat you at the facility where you are participating in the study? Will they pay for it? Federal regulations for research in the United States require that consent forms tell you whether some form of compensation for injury will be available, but it is up to the researchers to tell you exactly what that involves. For many studies, the informed consent form will say that treatment of any injuries or harms must be covered by your own medical insurance. In this type of situation, it may be relevant whether you have health insurance or what your coverage is (e.g., your deductible).

\section{What Are My Responsibilities?}

Apart from the ethics of pay-for-participation, there are some other issues you should consider before agreeing to participate in such studies. For instance, if a person has undergone one study in January with one experimental medication, then undergoes a different study in April with a different experimental medication, it may be hard for researchers to tell whether a side effect that occurred in April was from the first or the second medication, or perhaps the interaction between them. This would mean that any research data from the second study could potentially be meaningless. Research is valuable only when it provides valid answers to important scientific questions. Research subjects play a very important role in this process, and they also have certain responsibilities. Giving accurate information about your health status to the researchers is one responsibility, so is making all efforts to comply with the study protocol, such as taking the drugs according to the schedule, coming in for scheduled visits, and cooperating with other aspects of the study. 
Failure to carry out your responsibilities in these various ways can seriously hurt a study. If a participant has failed to follow the protocol, or has taken other experimental drugs without telling the researchers, then the study results may be based on tainted data. If too many patients fail to live up to their side of the bargain, the whole study might be in jeopardy. Researchers might draw incorrect conclusions, and reports in a medical journal or recommendations that doctors change their practice based on the study could have serious consequences for patient care.

\section{Conclusion}

We hope this overview of medical research, as well as its ethics and regulations, has given you a toolbox of ideas and questions to call upon when you are asked to participate in a research study. As we have emphasized in this chapter, you may feel unsure about what is being proposed and what the risks and benefits to you are, so it is most important that you have the ability to ask questions. This discussion comes back to the bedrock principles of research, which emphasize that informed consent is central and that participants must be free to decide, based on complete information, whether to participate. Only by gaining some understanding of the research process and the specific project in question can you make sure you are being treated appropriately. If this chapter empowers you to decide whether to participate in a research project and, if you do, if it has given you the knowledge you need to be involved with your eyes wide open, then it is a success.

Meslin EM, Schwartz PH. To be or not to be - a research subject. In: Kushner TK, editor. Surviving health care: a manual for patients and their families. Cambridge [England]:

Cambridge University Press; 2010. p. 146-62. http://www.cambridge.org/9780521744416 